\title{
Bayesian Inference for Health Inequality and Welfare Using Qualitative Data*
}

\author{
David Gunawan \\ School of Economics, University of New South Wales, Sydney, NSW 2052, Australia \\ david.gunawan@unsw.edu.au \\ William E. Griffiths \\ Department of Economics, University of Melbourne, Vic 3010, Australia \\ wegrif@unimelb.edu.au \\ Duangkamon Chotikapanich \\ Department of Econometrics and Business Statistics, Monash University, Caulfield East, Vic \\ 3145, Australia \\ duangkamon.chotikapanich@monash.edu
}

Oct 23, 2017

\begin{abstract}
We show how to use Bayesian inference to compare two ordinal categorical distributions commonly occurring with data on self-reported health status. Procedures for computing probabilities for first and second order stochastic dominance and S-dominance are described, along with methodology for obtaining posterior densities for health inequality indexes. The techniques are applied to four years of data on Australian self-reported health status.

JEL classification numbers: C11, I14, I31.
\end{abstract}

Keywords: Dominance probabilities; ordinal data; inequality indexes.

Corresponding Author

William Griffiths

Department of Economics

University of Melbourne 3010

Australia

Email: wegrif@unimelb.edu.au

* Comments from an anonymous reviewer have greatly improved the paper. 


\section{Introduction}

A general recognition that a society's well-being involves several dimensions has led to studies which measure levels of, and inequality in, income, education, health, and overall happiness. Obtaining a cardinal measure of at least two of these dimensions, health and overall happiness, is difficult and controversial. Less difficult, but also not without controversy, are ordinal measures of these two dimensions. Health status, for example, is often measured on a self-reported categorical scale. To assess whether there has been an improvement in the level of health, in health inequality, or in both, requires a comparison of two or more ordinal categorical distributions at different points in time. Similarly, for comparisons across countries or regions we need to compare the ordinal categorical distributions for those countries/regions. Several criteria have been suggested in the literature for comparing such distributions. Those that we focus on are:

1. First and second order stochastic dominance.

2. A spread dominance concept introduced by Allison and Foster (2004).

3. A measure of inequality proposed by Abul Naga and Yalcin (2008).

4. A measure of inequality proposed by Cowell and Flachaire (2017).

Comparisons using these criteria can be made directly using empirical distributions and the indices derived from them, aided by hypothesis tests and sampling theory distribution results where available. Yalonetzky (2013) has developed nonparametric sampling theory tests for first and second order stochastic dominance. Cowell and Flachaire (2017) develop asymptotic distribution theory for their proposed index.

In this paper we describe and illustrate Bayesian inference for comparing two ordinal categorical distributions, paying particular attention to the four listed criteria. What is novel about our Bayesian approach is the ability to report results in terms of posterior probabilities. For example, for two distributions $X$ and $Y$, we can report the probability that $X$ 
dominates $Y$, the probability that $Y$ dominates $X$, and the probability that neither distribution is dominant. Similarly, posterior distributions for the various indices can be used to make probability statements about their values.

In Section 2 the dominance and inequality criteria are described. In Section 3 we outline the Bayesian methodology for drawing inferences about these criteria. Section 4 contains an example, and concluding remarks are offered in Section 5.

\section{Dominance and Inequality Measures for Ordinal Data}

Consider a discrete random variable $X=\{1,2, \ldots, k\}$ whose values represent possible values on an ordered scale for self-assessed health or subjective well-being. It is assumed $X=k$ is the most favourable outcome and $X=1$ is the least favourable outcome. An example is selfassessed health on a scale where $X=1$ for poor, $X=2$ for fair, $X=3$ for good, $X=4$ for very good, and $X=5$ for excellent. Apart from being ordered, the values of $X$ are arbitrary with no cardinal values. Let the population proportion of individuals in category $j$ be given by $p_{X}(j)$, with $0 \leq p_{X}(j) \leq 1$, and $\sum_{j=1}^{k} p_{X}(j)=1$. The distribution function will be denoted by $F_{X}(j)=\sum_{i=1}^{j} p_{X}(i)$. Our focus is on univariate distributions such as health or well-being. There is a large literature on bivariate and multivariate distributions which relate variables such as health to income and other socio-economic measures. See, for example, many of the papers in Dias and O’Donnell (2013).

The first two criteria we consider are first and second order stochastic dominance. They are concerned with whether one distribution represents a higher level of well-being or an improvement in well-being relative to another. Given any two well-being distributions, $X$ and $Y$, with the same number of categories $k$, we say $X$ first order stochastic dominates $Y$, written as, $X>_{F S D} Y$, if and only if

$$
F_{X}(j) \leq F_{Y}(j) \text { for all } j \quad \text { and } F_{X}(j)<F_{Y}(j) \text { for some } j
$$


for $j=1,2, \ldots, k-1$. Here, distribution $X$ has a lower percentage of its population in the lowest $j$ categories, than distribution $Y$, and hence a higher average level of well-being for any increasing scale. Only $k-1$ categories need to be compared since the cumulative proportions of populations at the $k$-th category, $F_{X}(k)$ and $F_{Y}(k)$, are always equal to one. This condition for first order stochastic dominance was described by Allison and Foster (2004) and Yalonetzky (2013). Within the context of welfare functions that are additively separable and symmetric with respect to individuals, Yalonetzky shows that first order stochastic dominance implies a welfare improvement for the class of functions that are monotonically increasing. Also, if $X>_{F S D} Y$, the mean of $X$ is greater than the mean of $Y$ for all cardinal scalings of the categories.

Conditions for second order stochastic dominance of distribution $X$ over $Y$, written as $X>_{S S D} Y$, are

$$
\sum_{i=1}^{j} F_{X}(i) \leq \sum_{i=1}^{j} F_{Y}(i) \text { for all } j \quad \text { and } \quad \sum_{i=1}^{j} F_{X}(i)<\sum_{i=1}^{j} F_{Y}(i) \text { for some } j
$$

for $j=1,2, \ldots, k-1$. These conditions are presented by Yalonetzky (2013) who shows that $X>_{S S D} Y$ implies greater welfare for distribution $X$ relative to distribution $Y$ for the class of individual welfare functions which are both monotonically increasing and concave - welfare increases between successively higher ordinal categories are smaller.

A third criterion that we pursue is an inequality concept introduced by Allison and Foster (2004). For two distributions which have the same median, they define distribution $Y$ as having a greater spread than distribution $X$, written as $y S x$, if:

- $\quad$ Distributions $X$ and $Y$ have the same median category $m$,

- $\quad F_{X}(j) \leq F_{Y}(j)$ for all categories of $j<m$,

- $\quad F_{X}(j) \geq F_{Y}(j)$ for all categories of $j \geq m$. 
Distribution $X$ first order dominates $Y$ below the median category and $Y$ first order dominates $X$ for the median category and above. Using this measure, $y S x$ implies distribution $Y$ has greater inequality than distribution $X$. Since, for two distributions with the same median, greater inequality is considered less desirable, and to make dominance a favourable concept in line with first and second order stochastic dominance, we say that $X$ “equality dominates” $Y$, written $X \geq_{E D} Y$ if and only if $y S x$.

In addition to the dominance criteria, we also consider two indices that have been suggested for ordering ordinal categorical distributions, those developed by Abul Naga and Yalcin (2008) and Cowell and Flachaire (2017). Abul Naga and Yalcin develop an inequality index based on axioms of continuity, scale invariance, normalisation, and an aversion to greater inequality in the sense introduced by Allison and Foster (2004). Their measure is

$$
I_{X}(\alpha, \beta)=\frac{\sum_{j<m}\left(F_{X}(j)\right)^{\alpha}-\sum_{j \geq m}\left(F_{X}(j)\right)^{\beta}+(k+1-m)}{(m-1)(0.5)^{\alpha}-\left(1+(k-m)(0.5)^{\beta}\right)+(k+1-m)}
$$

where $\alpha$ and $\beta$ reflect value judgements of society, chosen by the analyst. When $\alpha=\beta=1$, the cumulative distributions for the lower half and upper half are given equal weight in the overall inequality. For a given value of $\beta$, as $\alpha \rightarrow \infty$, less weight is given to the inequality below the median, while for a given value of $\alpha$ and as $\beta \rightarrow \infty$, less weight is given to inequality above the median. When everyone is in the median category $I_{X}(1,1)=0$ and when half of the population is in the lowest category and the other half in the highest category, then $I_{X}(1,1)=1$

Using a generalized entropy index, Cowell and Flachaire (2017) consider inequality from the standpoint of an individual's perception of their status in the health distribution. Indices based on “downward looking” and "upward looking” assessment of health status and which satisfy several desirable axioms are developed. Costa-Font and Cowell (2013) use 
these measures to compute health inequality for several countries, and tentatively recommend the upward looking index

$$
U_{X}(\alpha)=\left\{\begin{array}{lr}
\frac{1}{\alpha(\alpha-1)}\left[\sum_{j=1}^{k} p_{X}(j)\left[s_{X}(j)\right]^{\alpha}-1\right] \quad \text { for } \alpha<1 \text { and } \alpha \neq 0 \\
-\sum_{j=1}^{k} p_{X}(j) \log s_{X}(j) & \text { for } \alpha=0
\end{array}\right.
$$

where $s_{X}(j)=\sum_{i=j}^{K} p_{X}(i)=1-F_{X}(j)+p_{X}(j)$. With perfect inequality where $s_{X}(j)=1$ for everybody, $U_{X}(\alpha)=0$. Otherwise, $U_{X}(\alpha)>0$. The parameter $\alpha$ governs the sensitivity of the index to particular parts of the distribution. A generalized entropy index has also been used by Madden (2010) in a study comparing ordinal and cardinal measures of inequality.

\section{Bayesian Inference}

In this section we describe the Bayesian methodology that can be used to make probability statements about the criteria specified in equations (1) to (5). Given a random sample of $n$ individuals each of whom allocates themselves to one and only one of $k$ ordinal categories, the vector containing the sample numbers of observations in each category, $\boldsymbol{n}^{\prime}=\left(n_{1}, n_{2}, \ldots, n_{k}\right)$, follows a multinomial distribution with density function

$$
f(\boldsymbol{n} \mid \boldsymbol{p}) \propto p_{1}^{n_{1}} p_{2}^{n_{2}} \cdots p_{k}^{n_{k}}
$$

In (6), $\sum_{j=1}^{k} n_{j}=n$, and the vector of parameters $\boldsymbol{p}^{\prime}=\left(p_{1}, p_{2}, \ldots, p_{k}\right)$ describes the proportions of the population that fall into each of the $k$ categories. Our objective is to use the sample observations $\boldsymbol{n}$ to first draw inferences about $\boldsymbol{p}$ and then, more importantly, to make inferences about the inequalities in equations (1) to (3), and the indexes in (4) and (5), all of which depend on $\boldsymbol{p}$. Within a Bayesian framework, the first step towards such inferences is the specification of a prior distribution for $\boldsymbol{p}$. Adopting the noninformative prior (Gelman et al 2014) 


$$
f(\boldsymbol{p}) \propto p_{1}^{-1} p_{2}^{-1} \ldots p_{k}^{-1}
$$

and combining it with the likelihood function in (6), we obtain the posterior density function for $\boldsymbol{p}$ as

$$
f(\boldsymbol{p} \mid \boldsymbol{n}) \propto f(\boldsymbol{n} \mid \boldsymbol{p}) f(\boldsymbol{p}) \propto p_{1}^{n_{1}-1} p_{2}^{n_{2}-1} \cdots p_{k}^{n_{k}-1}
$$

This density is a Dirichlet distribution with mean $E\left(p_{i} / \boldsymbol{n}\right)=n_{i} / n$ and covariance matrix

$$
\operatorname{cov}(\boldsymbol{p} \mid \boldsymbol{n})=\frac{1}{n^{2}(n+1)}\left[\begin{array}{cccc}
n_{1}\left(n-n_{1}\right) & -n_{1} n_{2} & \cdots & -n_{1} n_{k} \\
-n_{2} n_{1} & n_{2}\left(n-n_{2}\right) & \cdots & -n_{2} n_{k} \\
\vdots & \vdots & \ddots & \vdots \\
-n_{k} n_{1} & -n_{k} n_{2} & \cdots & n_{k}\left(n-n_{k}\right)
\end{array}\right]
$$

If interest centres on mean self-assessed health for a linear cardinal scaling $c_{i}$, say $\mu=\sum_{j=1}^{k} c_{j} j p_{j}=\sum_{j=1}^{k} d_{j} p_{j}$ where $d_{j}=c_{j} j$, then the posterior mean and variance of $\mu$ can be readily derived from the posterior mean and covariance for $\boldsymbol{p}$. Specifically, $E(\mu \mid \boldsymbol{n})=\sum_{j=1}^{K} d_{j} n_{j} / n \quad$ and $\quad \operatorname{var}(\mu \mid \boldsymbol{n})=\boldsymbol{d}^{\prime} \operatorname{cov}(\boldsymbol{p}) \boldsymbol{d} \quad$ where $\quad \boldsymbol{d}^{\prime}=\left(d_{1}, d_{2}, \ldots, d_{k}\right)$. More elaborate methods for cardinalisation have received attention in the literature. See, for example, van Doorslaer and Jones (2003) and Madden (2010).

For drawing inferences about $I_{X}(\alpha, \beta)$ and $U_{X}(\alpha)$, and the dominance criteria in (1), (2) and (3), which involve complex nonlinear functions of $\boldsymbol{p}$, closed form expressions are not available and we need to resort to simulation. Posterior densities for $I_{X}(\alpha, \beta)$ and $U_{X}(\alpha)$ can be estimated by obtaining draws $\boldsymbol{p}^{(m)}, m=1,2, \ldots, M$, from the Dirichlet posterior distribution for $\boldsymbol{p}$ and computing corresponding values of $I_{X}(\alpha, \beta)$ and $U_{X}(\alpha)$ for each 
draw. ${ }^{1}$ These values can be used to estimate the means and variances of the two indexes as well as to plot kernel density estimates of their posterior densities.

For each of the inequalities in (1), (2) and (3) we are concerned with the posterior probability that the relevant inequality holds. Uncertainty about whether an inequality holds arises because of uncertainty about $\boldsymbol{p}_{X}$ and $\boldsymbol{p}_{Y}$, the population proportions of individuals in each of the categories. This uncertainty can be quantified by drawing $\boldsymbol{p}_{X}$ and $\boldsymbol{p}_{Y}$ from their respective Dirichlet posterior density functions, and computing the proportions of the draws that satisfy each of the inequalities. These proportions are estimates of the probabilities that the inequalities hold. For example, given $M$ pairs of draws $\left(\boldsymbol{p}_{X}^{(m)}, \boldsymbol{p}_{Y}^{(m)}\right), m=1,2, \ldots, M$, the posterior probability that $X>_{F S D} Y$ can be estimated by computing $F_{X}^{(m)}(j)-F_{Y}^{(m)}(j)$ for each pair of draws, and counting the proportion of draws for which $F_{X}^{(m)}(j)-F_{Y}^{(m)}(j) \leq 0$ for all $j=1,2, \ldots, k-1$. That is,

$$
\operatorname{Pr}\left[X>_{F S D} Y\right]=\frac{1}{M} \sum_{m=1}^{M} \prod_{j=1}^{k-1} I\left[\left(F_{X}^{(m)}(j)-F_{Y}^{(m)}(j)\right) \leq 0\right]
$$

where $I[\bullet]$ is an indicator function equal to one if its argument is true and zero otherwise. ${ }^{2}$ Following similar procedures for equations (2) and (3), with the extra condition for (3) that the median categories for $X$ and $Y$ must be the same, yields probabilities for $X$ dominating $Y$ with respect to SSD and ED. Counting the proportions with the inequalities reversed, yields estimates for probabilities of $Y$ dominating $X$. The posterior probability that neither distribution dominates is given by $1-\operatorname{Pr}\left[X>_{F S D} Y\right]-\operatorname{Pr}\left[Y>_{F S D} X\right]$.

\footnotetext{
${ }^{1}$ To draw an observation $\boldsymbol{p}$ from a Dirichlet distribution with parameters $\boldsymbol{n}$, we draw observations $q_{i}, i=1,2, \ldots, k$ from $k$ independent gamma distributions with scale parameter 1 and shape parameters $n_{i}$ and compute $p_{i}=q_{i} / \sum_{i=1}^{k} q_{i}$

${ }^{2}$ Chotikapanich and Griffiths (2006) use a similar procedure to compute dominance probabilities for continuous distributions.
} 


\section{Example}

The data used to illustrate the methodology are the Self Reported Health Status (SRHS) obtained from the Household, Income, and Labour Dynamics in Australia (HILDA) survey for the years 2002, 2005, 2008, and 2010. The HILDA survey is a national representative longitudinal survey, which began in Australia in 2001; it is designed, managed, and maintained by the Melbourne Institute of Applied Economic and Social Research, University of Melbourne. Individuals aged 15 years or above answer a question of the form: in general, would you say your health is poor, fair, good, very good or excellent? Sample sizes are between 11000 to 12000 for each year being considered and the median category is good for all years. The numbers in each category and the empirical distribution functions are presented in Table 1 . They reveal negatively skewed distributions for all years; there are more people in the better health categories and less people in the inferior health categories.

Table 1: Sample Sizes and Cumulative Proportions for Categorical Self-Reported Health Status Distributions

\begin{tabular}{crrrrrrrr}
\hline & \multicolumn{2}{c}{2002} & \multicolumn{2}{c}{2005} & \multicolumn{2}{c}{2008} & \multicolumn{2}{c}{2010} \\
Category & $n$ & prop & $n$ & prop & $n$ & prop & $n$ & prop \\
\hline Poor & 381 & 0.0323 & 362 & 0.0320 & 318 & 0.0286 & 359 & 0.0300 \\
Fair & 1626 & 0.1700 & 1644 & 0.1771 & 1576 & 0.1705 & 1722 & 0.1741 \\
Good & 4154 & 0.5219 & 4116 & 0.5404 & 4063 & 0.5363 & 4337 & 0.5369 \\
Very good & 4218 & 0.8792 & 4037 & 0.8967 & 3931 & 0.8902 & 4265 & 0.8937 \\
Excellent & 1426 & 1.0000 & 1169 & 1.0000 & 1220 & 1.0000 & 1271 & 1.0000 \\
Sample size & 11805 & & 11328 & & 11108 & & 11954 & \\
\hline
\end{tabular}

Posterior means and standard deviations for the population proportions, given by $n_{i} / n$ and $\left[n_{i}\left(n-n_{i}\right) /\left(n^{2}(n+1)\right)\right]^{0.5}$, respectively, are reported in Table 2. For estimating posterior probabilities of dominance and posterior densities of the inequality measures, we draw $M=10000$ independent draws from each of the Dirichlet posterior distributions. 
Table 2: Posterior means and standard deviations for the population proportions of the SRHS

\begin{tabular}{lccccc}
\hline Category & & 2002 & 2005 & 2008 & 2010 \\
\hline Poor & $p_{1}$ & 0.0323 & 0.0320 & 0.0286 & 0.0300 \\
& & $(0.0016)$ & $(0.0017)$ & $(0.0016)$ & $(0.0016)$ \\
\multirow{2}{*}{ Fair } & $p_{2}$ & 0.1377 & 0.1451 & 0.1419 & 0.1441 \\
& & $(0.0032)$ & $(0.0033)$ & $(0.0033)$ & $(0.0032)$ \\
Good & $p_{3}$ & 0.3519 & 0.3633 & 0.3658 & 0.3628 \\
& & $(0.0044)$ & $(0.0045)$ & $(0.0046)$ & $(0.0044)$ \\
Very good & $p_{4}$ & 0.3573 & 0.3564 & 0.3539 & 0.3568 \\
\multirow{2}{*}{ Excellent } & & $(0.0044)$ & $(0.0045)$ & $(0.0045)$ & $(0.0044)$ \\
& $p_{5}$ & 0.1208 & 0.1032 & 0.1098 & 0.1063 \\
& & $(0.0030)$ & $(0.0029)$ & $(0.0030)$ & $0.0028)$ \\
\hline
\end{tabular}

The posterior probabilities for FSD are reported in Tables 3 for the 6 possible pairwise comparisons of the 4 years. The numbers in parentheses are numerical standard errors calculated as $[\operatorname{prob}(1-\operatorname{prob}) / 10000]^{1 / 2}$. With the exception of the $2005 / 2008$ comparison, the probabilities for no dominance are always greater than 0.5. There are, however, several probabilities of dominance that are moderately large in the sense that they are greater than 0.33, namely, (2002 $\left.>_{F S D} 2005\right),\left(2008>_{F S D} 2005\right),\left(2010>_{F S D} 2005\right)$ and $\left(2008>_{F S D} 2010\right)$. These results suggest that the self-assessed health distribution in 2005 was a poor one. This was the year in which there were substantial changes in Medicare rebates, leading to increases in prices of a number of medical services. It seems likely that the uncertainty created by these changes has led to a perception of a decline in health status. Comparing 2002 with 2010, we find the probability of no dominance is 0.84 . Thus, there is little evidence to suggest that the distribution has improved over time.

Because FSD implies SSD, the posterior probabilities for SSD will be at least as great as the corresponding ones for FSD. The posterior probabilities for SSD reported in Table 4 generally support the conclusions about the SRHS distributions that were drawn from the FSD results, but because the dominance probabilities are larger, there is more evidence of dominance. Three of the pairwise comparisons whose FSD probabilities were greater than 
0.33 all have SSD probabilities greater than 0.5 , and there is evidence of worse perceptions about health in 2005 than in other years.

Table 3: Probabilities (numerical standard errors) for First Order Stochastic Dominance

\begin{tabular}{|c|c|c|c|c|c|c|}
\hline & $\begin{array}{cc}02 & 05 \\
A & B\end{array}$ & $\begin{array}{cc}02 & 08 \\
A & B\end{array}$ & $\begin{array}{cc}02 & 10 \\
A & B\end{array}$ & $\begin{array}{cc}05 & 08 \\
A & B\end{array}$ & $\begin{array}{cc}05 & 10 \\
A & B\end{array}$ & $\begin{array}{cc}08 & 10 \\
A & B\end{array}$ \\
\hline $\operatorname{Pr}\left(A>_{F S D} B\right)$ & $\begin{array}{l}0.4274 \\
(0.0016)\end{array}$ & $\begin{array}{l}0.0449 \\
(0.0007)\end{array}$ & $\begin{array}{l}0.1503 \\
(0.0011)\end{array}$ & $\begin{array}{l}0.0014 \\
(0.0001)\end{array}$ & $\begin{array}{l}0.0171 \\
(0.0004)\end{array}$ & $\begin{array}{l}0.3306 \\
(0.0015)\end{array}$ \\
\hline $\operatorname{Pr}\left(B>_{F S D} A\right)$ & $\begin{array}{l}0.0000 \\
(0.0000)\end{array}$ & $\begin{array}{l}0.0004 \\
(0.0000)\end{array}$ & $\begin{array}{l}0.0000 \\
(0.0000)\end{array}$ & $\begin{array}{l}0.6345 \\
(0.0015)\end{array}$ & $\begin{array}{l}0.4136 \\
(0.0016)\end{array}$ & $\begin{array}{l}0.0237 \\
(0.0005)\end{array}$ \\
\hline $\operatorname{Pr}($ no dominance) & $\begin{array}{l}0.5726 \\
(0.0016)\end{array}$ & $\begin{array}{l}0.9547 \\
(0.0007)\end{array}$ & $\begin{array}{l}0.8497 \\
(0.0011)\end{array}$ & $\begin{array}{l}0.3641 \\
(0.0015)\end{array}$ & $\begin{array}{l}0.5693 \\
(0.0016)\end{array}$ & $\begin{array}{l}0.6457 \\
(0.0015)\end{array}$ \\
\hline
\end{tabular}

Table 4: Probabilities (numerical standard errors) for Second Order Stochastic Dominance

\begin{tabular}{|c|c|c|c|c|c|c|}
\hline & $\begin{array}{cc}02 & 05 \\
A & B\end{array}$ & $\begin{array}{cc}02 & 08 \\
A & B\end{array}$ & $\begin{array}{cc}02 & 10 \\
A & B\end{array}$ & $\begin{array}{cc}05 & 08 \\
A & B\end{array}$ & $\begin{array}{cc}05 & 10 \\
A & B\end{array}$ & $\begin{array}{cc}08 & 10 \\
A & B\end{array}$ \\
\hline $\operatorname{Pr}\left(A>_{S S D} B\right)$ & $\begin{array}{l}0.4330 \\
(0.0016)\end{array}$ & $\begin{array}{l}0.0473 \\
(0.0007)\end{array}$ & $\begin{array}{l}0.1534 \\
(0.0011)\end{array}$ & $\begin{array}{l}0.0108 \\
(0.0003)\end{array}$ & $\begin{array}{l}0.0607 \\
(0.0008)\end{array}$ & $\begin{array}{l}0.5506 \\
(0.0016)\end{array}$ \\
\hline $\operatorname{Pr}\left(B>_{S S D} A\right)$ & $\begin{array}{l}0.0004 \\
(0.0000)\end{array}$ & $\begin{array}{l}0.0414 \\
(0.0006)\end{array}$ & $\begin{array}{l}0.0065 \\
(0.0003)\end{array}$ & $\begin{array}{l}0.8434 \\
(0.0011)\end{array}$ & $\begin{array}{l}0.6306 \\
(0.0015)\end{array}$ & $\begin{array}{l}0.0861 \\
(0.0009)\end{array}$ \\
\hline $\operatorname{Pr}($ no dominance) & $\begin{array}{l}0.5666 \\
(0.0016)\end{array}$ & $\begin{array}{l}0.9113 \\
(0.0009)\end{array}$ & $\begin{array}{l}0.8401 \\
(0.0012)\end{array}$ & $\begin{array}{l}0.1458 \\
(0.0011)\end{array}$ & $\begin{array}{l}0.3087 \\
(0.0015)\end{array}$ & $\begin{array}{l}0.3633 \\
(0.0015)\end{array}$ \\
\hline
\end{tabular}

Turning to the equality dominance probabilities in Table 5, we find little evidence of any dominance. $\operatorname{Pr}\left(2008>_{E D} 2002\right)=0.43$ and $\operatorname{Pr}\left(2010>_{E D} 2002\right)=0.19$, but all other dominance probabilities are less than 0.07 . More evidence of greater inequality in 2002 is revealed by examining results from the Abul Naga/Yalcin and Cowell/Flachaire indexes. Their posterior means and standard deviations are presented in Table 6. For the Abul Naga/Yalcin index we used $(\alpha=1, \beta=1), \quad(\alpha=1, \beta=4)$ and $(\alpha=4, \beta=1)$; for the Cowell/Flachaire index we set $\alpha=0.1$ and $\alpha=0.9$. Posterior densities for the Abul Naga/Yalcin index for $(\alpha=1, \beta=1)$ are graphed in Figure 1; for the Cowell/Flachaire index posterior densities were graphed for both settings of $\alpha$ and presented as Figure 2(a) and (b). The posterior means and the complete densities in the figures all lead to the same conclusion. 
Inequality is similar in 2005, 2008 and 2010, but greater in 2002. Just prior to 2002, the government introduced major reforms to private health insurance. A possible consequence of these reforms is greater inequality in health perceptions.

Table 5: Probabilities (numerical standard errors) for Equality Dominance

\begin{tabular}{|c|c|c|c|c|c|c|}
\hline & $\begin{array}{cc}02 & 05 \\
A & B \\
\end{array}$ & $\begin{array}{cc}02 & 08 \\
A & B \\
\end{array}$ & $\begin{array}{cc}02 & 10 \\
A & B \\
\end{array}$ & $\begin{array}{cc}05 & 08 \\
A & B \\
\end{array}$ & $\begin{array}{cc}05 & 10 \\
A & B\end{array}$ & $\begin{array}{cc}08 & 10 \\
A & B\end{array}$ \\
\hline $\operatorname{Pr}\left(A>_{E D} B\right)$ & $\begin{array}{l}0.0000 \\
(0.0000)\end{array}$ & $\begin{array}{l}0.0000 \\
(0.0000)\end{array}$ & $\begin{array}{l}0.0000 \\
(0.0000)\end{array}$ & $\begin{array}{l}0.0083 \\
(0.0003)\end{array}$ & $\begin{array}{l}0.0371 \\
(0.0006)\end{array}$ & $\begin{array}{l}0.0616 \\
(0.0008)\end{array}$ \\
\hline $\operatorname{Pr}\left(B>_{E D} A\right)$ & $\begin{array}{l}0.0635 \\
(0.0008)\end{array}$ & $\begin{array}{l}0.4349 \\
(0.0016)\end{array}$ & $\begin{array}{l}0.1864 \\
(0.0012)\end{array}$ & $\begin{array}{l}0.0208 \\
(0.0005)\end{array}$ & $\begin{array}{l}0.0462 \\
(0.0007)\end{array}$ & $\begin{array}{l}0.0266 \\
(0.0005)\end{array}$ \\
\hline $\operatorname{Pr}($ no dominance) & $\begin{array}{l}0.9365 \\
(0.0008)\end{array}$ & $\begin{array}{l}0.5651 \\
(0.0016)\end{array}$ & $\begin{array}{l}0.8136 \\
(0.0012)\end{array}$ & $\begin{array}{l}0.9709 \\
(0.0005)\end{array}$ & $\begin{array}{l}0.9167 \\
(0.0009)\end{array}$ & $\begin{array}{l}0.9118 \\
(0.0009)\end{array}$ \\
\hline
\end{tabular}

Table 6: Inequality measures, 2002-2010

\begin{tabular}{cccccc}
\hline & \multicolumn{3}{c}{ Abul Naga and Yalcin Index } & \multicolumn{2}{c}{ Cowell and Flachaire Index } \\
& $\alpha=1, \beta=1$ & $\alpha=1, \beta=4$ & $\alpha=4, \beta=1$ & $\alpha=0.1$ & $\alpha=0.9$ \\
\hline 2002 & 0.4006 & 0.5324 & 0.5331 & 0.6153 & 3.7164 \\
& $(0.00314)$ & $(0.00326)$ & $(0.00565)$ & $(0.00176)$ & $(0.01049)$ \\
2005 & 0.3859 & 0.5137 & 0.5011 & 0.6122 & 3.6866 \\
& $(0.00313)$ & $(0.00337)$ & $(0.00561)$ & $(0.00195)$ & $(0.01099)$ \\
2008 & 0.3863 & 0.5177 & 0.5106 & 0.6125 & 3.6859 \\
& $(0.00318)$ & $(0.00339)$ & $(0.00571)$ & $(0.00188)$ & $(0.01101)$ \\
2010 & 0.3868 & 0.5158 & 0.5070 & 0.6121 & 3.6832 \\
& $(0.00306)$ & $(0.00325)$ & $(0.00547)$ & $(0.00187)$ & $(0.01066)$ \\
\hline
\end{tabular}

\section{Concluding Remarks}

Various measures for comparing ordinal categorical distributions have been suggested in the literature for assessing improvements in welfare resulting from an increase in the level, and/or a decrease in inequality, of self-assessed health distributions. Bayesian inference is a convenient and straightforward method for providing probabilistic information on welfare improvements. We have demonstrated how to compute posterior probabilities of first and second order stochastic dominance, and equality dominance, and how to find posterior densities for indices designed to measure inequality. 


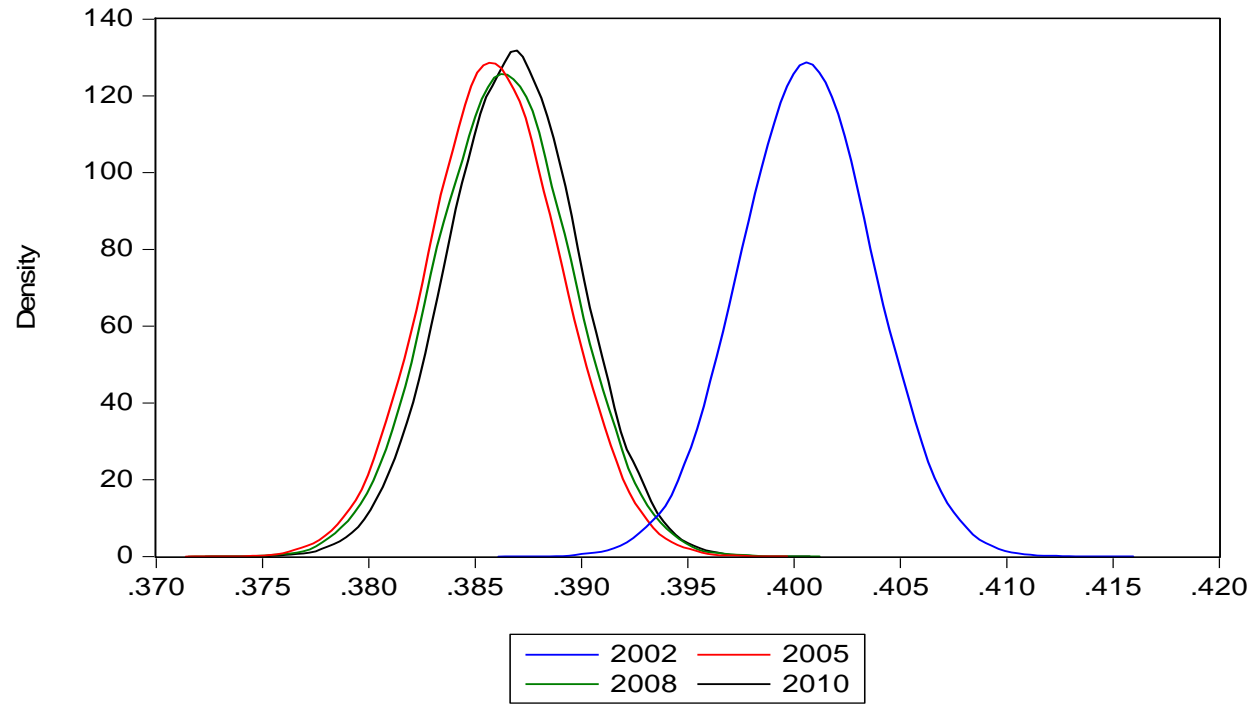

Figure 1 Posterior Densities for Abul Naga/Yalcin Index, $\alpha=1, \beta=1$

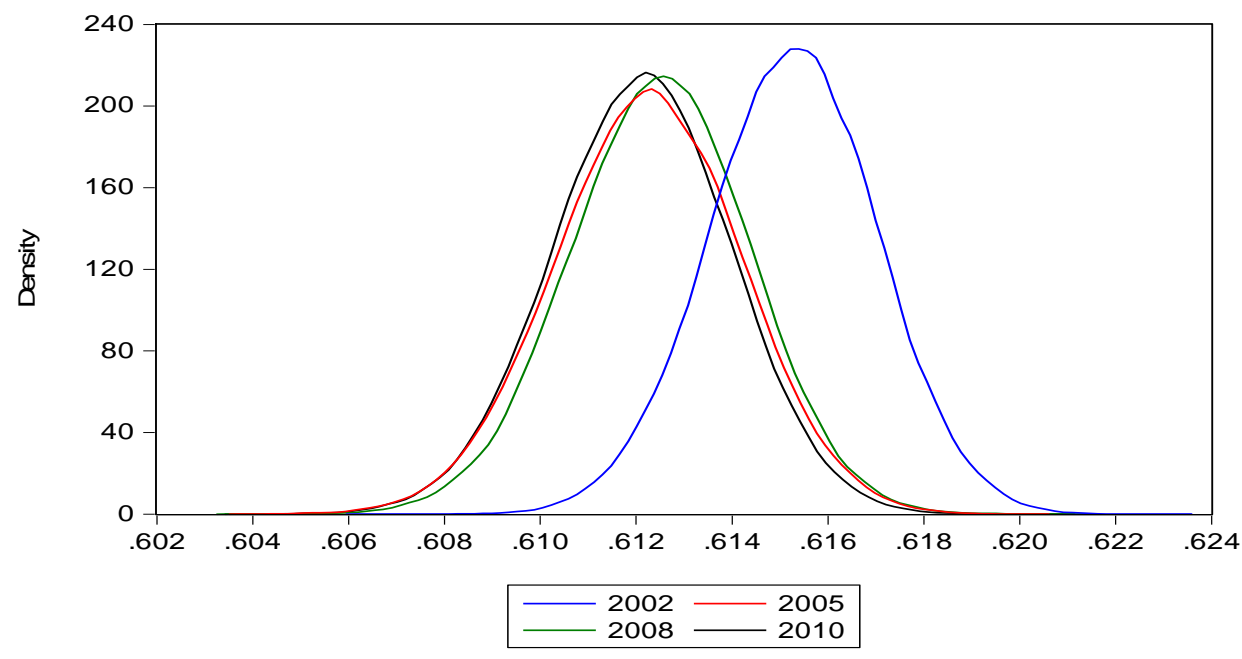

Figure 2(a) Posterior densities for Cowell/Flachaire Index $\alpha=0.1$

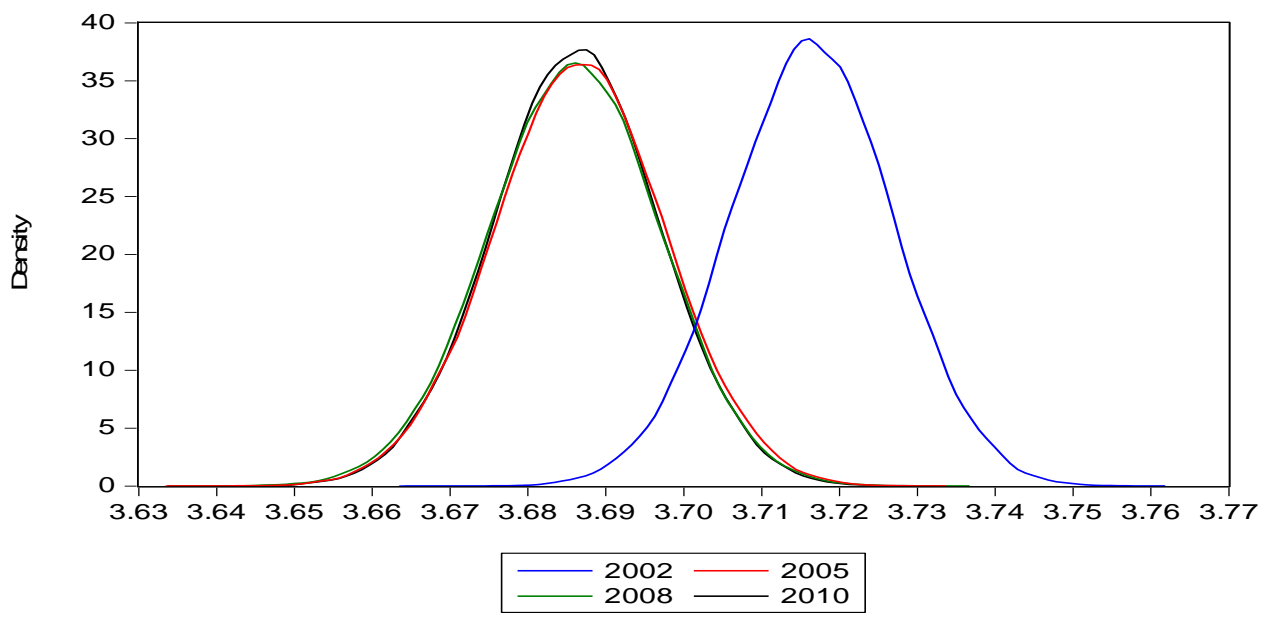

Figure 2(b) Posterior densities for Cowell/Flachaire Index $\alpha=0.9$ 


\section{References}

Abul Naga, R. H. and Yalcin, T (2008), "Inequality Measurement for Ordered Response Health Data”, Journal of Health Economics, 27, 1614-1625.

Allison, R. A. and Foster, J. E. (2004), “Measuring Health Inequality using Qualitative Data”, Journal of Health Economics, 23, 505-524.

Chotikapanich, D. and Griffiths, W.E. (2006), "Bayesian Assessment of Lorenz and Stochastic Dominance in Income Distributions”, in J. Creedy and G.Kalb, editors, Research on Economic Inequality, Volume 13: Dynamics of Inequality and Poverty, Amsterdam: Elsevier, 297-321.

Costa-Font, J. and Cowell, F. (2013), “Measuring Health Inequality with Categorical Data: Some Regional Patterns”. In P. R. Dias and O. O’Donnell, editors, Research on Economic Inequality, Volume 21: Health and Inequality, Amsterdam: Elsevier, 53-76.

Cowell, F. and Flachaire, E. (2017), "Inequality with Ordinal Data”, Economica, 84, 290321.

Dias, P.R. and O’Donnell, O. (2013), Research on Economic Inequality, Volume 21: Health and Inequality, Amsterdam: Elsevier, 53-76.

Gelman, A., Carlin, J. B., Stern, H. S., and Rubin, D. B. (1995), Bayesian Data Analysis, Chapman \& Hall.

Madden, D. (2010), Ordinal and Cardinal Measures of Health Inequality: An Empirical Comparison”, Health Economics Letters, 19, 243-250.

van Doorslaer, E. and Jones, A.M. (2003), "Inequalities in self-reported health: validation of a new approach to measurement”, Journal of Health Economics, 22, 61-87.

Yalonetzky, G. (2013), “Stochastic Dominance with Ordinal Variables: Conditions and a Test”, Econometric Reviews, 32:1, 126-163. 